\title{
THE SQUARE ROOT OF A POSITIVE SELF-ADJOINT OPERATOR
}

\author{
S. J. BERNAU
}

(Received 6 May 1966, revised 24 February 1967)

\section{Introduction}

One elementary proof of the spectral theorem for bounded self-adjoint operators depends on an elementary construction for the square root of a bounded positive self-adjoint operator. The purpose of this paper is to give an elementary construction for the unbounded case and to deduce the spectral theorem for unbounded self-adjoint operators. In so far as all our results are more or less immediate consequences of the spectral theorem there is little that is entirely new. On the other hand the elementary approach seems to the author to provide a deeper insight into the structure of the problem and also leads directly to the spectral theorem without appealing first to the bounded case. Besides this, our methods for proving uniqueness of the square root and of the spectral family seem to be new even in the bounded case. In particular there is no need to invoke representation theorems for linear functionals on spaces of continuous functions.

For positive self-adjoint $T$ and positive real $\lambda,(\lambda I+T)^{-1}$ is a bounded positive self-adjoint operator. Write $Y_{\lambda}=\left\{(\lambda I+T)^{-1}\right\}^{\frac{1}{2}}$ and $B_{\lambda}=(\lambda I+T) Y_{\lambda}$. Letting $\lambda \rightarrow 0+0$ we obtain an operator $B$ which is positive self-adjoint and $B^{2}=T$.

After obtaining the square root we follow the approach of $[4, \S 108]$ to obtain for self-adjoint $A$, the 'Jordan decomposition' $A=A^{+}-A^{-}$with $A^{+}$and $A^{-}$self-adjoint and positive and the range of each contained in the null space of the other. The spectral projections are then given, as in the bounded case, by defining $E(\lambda)$ to be the orthogonal projection on the null space of $(A-\lambda I)+$ for all real $\lambda$.

The contents of this paper apart from Theorems 19 and 20 form the basic material on which [1] depends. For completeness some standard results about unbounded operators are also included. These form most of $\S 2$.

Added 22 February 1967. An earlier version of this paper was submitted to Proc. London Math. Soc. in March 1965. Since that time a shorter elementary proof of the existence of the square root of a positive operator 
has been obtained independently by A. Wouk [6]. Wouk's method is different from the method of this paper and some details are suppressed. $\mathrm{He}$ does not obtain the full strength of Theorem 10 although there is no difficulty in proving Theorem 10 from his construction; nor does he give a uniqueness proof.

I am grateful to the referee for his helpful comments on this paper.

\section{Definitions and preliminary results}

Let $\mathfrak{S}$ be a Hilbert space, real or complex, and let $T$ be a linear operator whose domain $\mathfrak{D}(T)$ and range $\Re(T)$ are subspaces of $\mathfrak{H}$. The Cartesian product $\mathfrak{S C} \times \mathfrak{F}$ is a Hilbert space under natural linear operations and the inner product

$$
\left(\left[x_{1}, y_{1}\right],\left[x_{2}, y_{2}\right]\right)=\left(x_{1}, x_{2}\right)+\left(y_{1}, y_{2}\right) \quad\left(x_{1}, x_{2}, y_{1}, y_{2} \in \mathfrak{S}\right) .
$$

The graph of $T, \Gamma(T)$ is the set $\{[x, T x]: x \in \mathscr{D}(T)\} ; \Gamma(T)$ is a subspace of $\mathfrak{S} \times \mathfrak{S}$. $T$ is closed if, whenever $\left(x_{n}\right)$ is a sequence of elements of $\mathfrak{D}(T)$ such that $x_{n} \rightarrow x$ and $T x_{n} \rightarrow y$ then $x \in \mathscr{D}(T)$ and $T x=y$; equivalently $T$ is closed if $\Gamma(T)$ is closed in $\mathfrak{K} \times \mathfrak{S}$.

Suppose $\mathfrak{D}(T)$ is dense in $\mathfrak{S}$. We define an operator $T^{*}$ as follows: $\mathfrak{D}\left(T^{*}\right)$ is the set of $y$ in $\mathfrak{S}$ such that there exists $y^{*}$ in $\mathfrak{S}$ with the property

$$
(T x, y)=\left(x, y^{*}\right) \quad(x \in \mathfrak{D}(T))
$$

then, $T^{*} y=y^{*}\left(y \in \mathscr{D}\left(T^{*}\right)\right)$. $T^{*}$ is called the adjoint of $T$, it is uniquely defined and is a closed linear operator.

Throughout this paper we adopt the

Conventions. (i) All operators are linear with domain and range in $\mathfrak{H}$;

(ii) if $T$ is an operator any reference to $T^{*}$ implies that $\mathscr{D}(T)$ is dense in $\mathfrak{S}$ and any reference to $T$ being bounded implies that $\mathscr{D}(T)=\mathfrak{F}$;

(iii) the statement, $\left(x_{n}\right)$ is a sequence in $\mathfrak{D}(T)$, means, $\left(x_{n}\right)$ is a sequence of elements of $\mathfrak{D}(T)$.

Let $S$ and $T$ be operators; $S$ is an extension of $T$, written $S \supseteqq T$ or $T \subseteq S$, means that $\mathfrak{D}(T) \subseteq \mathfrak{D}(S)$, and $T x=S x(x \in \mathfrak{D}(T)) . T=S$ if and only if $T \subseteq S$ and $S \cong T$. If $T \subseteq S$ then $T^{*} \supseteqq S^{*}$. In general $T$ commutes with $S$ means $T S=S T$, however, if one of $S$ and $T$, say $S$, is bounded, $T$ commutes with $S$ means $S T \cong T S$.

$T$ is symmetric if $\mathfrak{D}(T)$ is dense in $\mathfrak{H}$ and

$$
(T x, y)=(x, T y) \quad(x, y \in \mathfrak{D}(T)) ;
$$

equivalently, $T$ is symmetric if $T \subseteq T^{*}$ (if $T \subseteq T^{*}$, Convention (ii) implies that $\mathfrak{D}(T)$ is dense). $T$ is self-adjoint if $T=T^{*} . T$ is positive if $T$ is selfadjoint and $(T x, x) \geqq 0(x \in \mathfrak{D}(T))$. 
For general properties of unbounded operators we refer to $[4, \S \S 114-119]$, and [2, Chapter XII, $\S 1]$. Results for which a reference is not given can be found in one of these places.

LEMMA 1. If $T$ is an operator with $\mathfrak{D}(T)$ dense in $\mathfrak{S}$, then $T$ has a closed linear extension if and only if $\mathfrak{D}\left(T^{*}\right)$ is dense in $\mathfrak{H}$. In this case $T^{* *}$ is the minimal closed extension of $T$ and $\Gamma\left(T^{* *}\right)$ is the closure, in $\mathfrak{F} \times \mathfrak{K}$, of $\Gamma(T)$. In particular, if $T$ is closed and $\mathscr{D}(T)$ is dense, $\mathscr{D}\left(T^{*}\right)$ is dense and $T=T^{* *}$.

This lemma is proved in $[4, \S 117]$, except for the statement about $\Gamma\left(T^{* *}\right)$. This statement is an easy deduction.

Lemma 2. Let $T$ be a closed operator with dense domain. Then,

(i) $\left(I+T^{*} T\right)^{-1}$ exists and is a bounded self-adjoint operator;

(ii) $T^{*} T$ is self-adjoint and positive;

(iii) if $T^{\prime}$ is the restriction of $T$ to $\mathfrak{D}\left(T^{*} T\right)$, then $\Gamma\left(T^{\prime}\right)$ is dense in $\Gamma(T)$.

This lemma is most of [2, Lemma XII 7.1]. Parts (i) and (ii) are also proved in $[4, \S \S 118,119]$.

As a corollary of Lemma 2 we have

LEMMA 3. If $T$ is a closed symmetric operator such that $T^{2}$ is an extension of a self-adjoint operator $S$ then $T$ is self-adjoint and $T^{2}=S$.

Proof. By Lemmas 1 and $2, T T^{*}=T^{* *} T^{*}$ is self-adjoint. Because $T$ is symmetric $T T^{*} \supseteqq T^{2}$ and, because $T^{2} \supseteq S$, and $S$ is self-adjoint, $T T^{*}=T^{2}=S$. Let $x \in \mathfrak{D}\left(T^{*}\right)$; by Lemma 2 (iii), there exists a sequence $\left(x_{n}\right)$ in $\mathfrak{D}\left(T T^{*}\right)=\mathfrak{D}\left(T^{2}\right)$ such that $x_{n} \rightarrow x$ and $T^{*} x_{n} \rightarrow T^{*} x$. Because $T$ is symmetric and $x_{n} \in \mathscr{D}(T), T^{*} x_{n}=T x_{n}$. Because $T$ is closed $x \in \mathfrak{D}(T)$ and

$$
T x=\lim T x_{n}=\lim T^{*} x_{n}=T^{*} x .
$$

Hence $T=T^{*}$ as required.

THEOREM 4. Let $T$ be a bounded positive self-adjoint operator. There exists a unique bounded positive self-adjoint operator $T^{\frac{1}{2}}$ such that $\left(T^{\frac{1}{2}}\right)^{2}=T$. $T^{\frac{1}{2}}$ can be obtained as the strong limit of a sequence of polynomials in $T$ and hence $T^{\frac{1}{2}}$ commutes with every bounded operator which commutes with $T$.

This theorem is proved in $[4, \S 104]$. We deduce

LEMMA 5. Let $T$ be a bounded positive self-adjoint operator and let $B$ a closed operator which commutes with $T$. Then, $B$ commutes with $T^{\frac{1}{2}}$.

Proof. Let $\left(p_{n}\right)$ be a sequence of polynomials in $T$ such $p_{n} x \rightarrow T^{\frac{1}{2}} x$ $(n \rightarrow \infty)(x \in \mathfrak{S})$ and let $x \in \mathfrak{D}(B)$. Clearly

hence

$$
p_{n} B \cong B p_{n} \quad(n=1,2, \cdots),
$$

and

$$
p_{n} x \in \mathfrak{D}(B)
$$




$$
p_{n} B x=B p_{n} x \quad(n=1,2, \cdots) .
$$

Now $p_{n} x \rightarrow T^{\frac{1}{2}} x$ and $B p_{n} x=p_{n} B x \rightarrow T^{\frac{1}{2}} B x(n \rightarrow \infty)$. Hence, because $B$ is closed, $T^{\frac{1}{2}} x \in \mathscr{D}(B)$ and $B T^{\frac{1}{2}} x=\lim B p_{n} x=T^{\frac{1}{2}} B x$. Thus $T^{\frac{1}{2}} B \subseteq B T^{\frac{1}{2}}$ as required.

\section{Unbounded positive operators}

Throughout this section $T$ denotes a, not necessarily bounded, positive self adjoint operator.

LeMma 6. Let $\lambda$ be a positive real number; then $(\lambda I+T)^{-1}$ exists and is a bounded positive self-adjoint operator.

Proof. If $x \in \mathfrak{D}(T)$,

$$
\begin{aligned}
\|(\lambda I+T) x\|^{2} & =\lambda^{2}\|x\|^{2}+2 \lambda(T x, x)+\|T x\|^{2} \\
& \geqq \lambda^{2}\|x\|^{2} .
\end{aligned}
$$

The remainder of the proof is omitted.

For the next two lemmas, I am very grateful to the referee of an earlier version of this paper for greatly simplifying my original arguments.

LEMMA 7. Let $\lambda$ and $\mu$ be positive real numbers, define

$$
Y_{\lambda}=\left\{(\lambda I+T)^{-1}\right\}^{\frac{1}{2}}, \quad B_{\lambda}=Y_{\lambda}^{-1} .
$$

Then, $B_{\lambda}$ is self-adjoint and positive, $B_{\lambda}^{2}=\lambda I+T, B_{\lambda}=(\lambda I+T) Y_{\lambda}$ and

$$
\mathfrak{D}\left(B_{\lambda}\right)=\mathfrak{R}\left(Y_{\lambda}\right)=\mathfrak{R}\left(Y_{\mu}\right)=\mathfrak{D}\left(B_{\mu}\right) .
$$

Proof. Note first that if $Y_{\lambda} x=0$,

$$
x=(\lambda I+T) Y_{\lambda}^{2} x=0
$$

so that $Y_{\lambda}^{-1}$ exists. Because $\Re\left(Y_{\lambda}\right) \supseteq \mathfrak{D}(\lambda I+T), \mathfrak{D}\left(B_{\lambda}\right)$ is dense and

$$
B_{\lambda}^{*}=\left(Y_{\lambda_{4}}^{-1}\right)^{*}=\left(Y_{\lambda}^{*}\right)^{-1}=Y_{\lambda}^{-1}=B_{\lambda} .
$$

Because $Y_{\lambda}^{2}$ commutes with $\lambda I+T$, Lemma 5 shows that

$$
Y_{\lambda}(\lambda I+T) \subseteq(\lambda I+T) Y_{\lambda}
$$

Hence,

and it follows that

$$
Y_{\lambda}(\lambda I+T) Y_{\lambda} \subseteq(\lambda I+T) Y_{\lambda}^{2}=I
$$

$$
B_{\lambda}=(\lambda I+T) Y_{\lambda} .
$$

If $x \in \mathfrak{D}\left(B_{\lambda}\right), x=Y_{\lambda} u$ for some $u$ in $\mathfrak{S}$ and

$$
\left(B_{\lambda} x, x\right)=\left(B_{\lambda} Y_{\lambda} u, Y_{\lambda} u\right)=\left(u, Y_{\lambda} u\right) \geqq 0 .
$$


Thus $B_{\lambda}$ is positive. Furthermore,

$$
B_{\lambda}^{2}=Y_{\lambda}^{-2}=\left(Y_{\lambda}^{2}\right)^{-1}=\lambda I+T .
$$

To see that $\Re\left(Y_{\lambda}\right)=\Re\left(Y_{\mu}\right)$ we observe that, because

$$
\begin{aligned}
\mathfrak{D}(\mu I+T) & =\mathfrak{D}(\lambda I+T)=\Re\left(Y_{\lambda}^{2}\right), \\
Y_{\mu}^{2}(\mu I+T) Y_{\lambda}^{2}=Y_{\lambda}^{2} & =(\mu I+T) Y_{\mu}^{2} Y_{\lambda}^{2}=(\mu I+T) Y_{\lambda}^{2} Y_{\mu}^{2} .
\end{aligned}
$$

It follows from (1) that $\left\|Y_{\lambda}^{2}\right\|=\left\|(\lambda I+T)^{-1}\right\| \leqq \lambda^{-1}$ so that $0 \leqq \lambda Y_{\lambda}^{2} \leqq I$ in the usual ordering of bounded self-adjoint operators. Because $T Y_{\lambda}^{2}=I-\lambda Y_{\lambda}^{2}$, it follows that $T Y_{\lambda}^{2}$ is bounded and $0 \leqq T Y_{\lambda}^{2} \leqq I$. Then, for $\mu>0$, $(\mu I+T) Y_{\lambda}^{2}$ is bounded self-adjoint and positive and commutes with $Y_{\mu}^{2}$. It now follows from Theorem 4 that

$$
Y_{\lambda}=Y_{\mu}\left\{(\mu I+T) Y_{\lambda}^{2}\right\}^{\frac{1}{2}}, \quad Y_{\mu}=Y_{\lambda}\left\{(\lambda I+T) Y_{\mu}^{2}\right\}^{\frac{1}{2}}
$$

and hence $\mathfrak{R}\left(Y_{\lambda}\right)=\Re\left(Y_{\mu}\right)$ as required.

LEMMA 8. $\mathfrak{D}\left(B_{\lambda} B_{\mu}\right)=\mathfrak{D}(T), B_{\lambda} B_{\mu}=B_{\mu} B_{\lambda} ;$ and, if $x \in \mathfrak{D}(T)$ and $0<\lambda<\mu$,

$$
\left(B_{\lambda} B_{\mu} x, x\right) \geqq\left(B_{\lambda}^{2} x, x\right) .
$$

Proof. Because $\mathfrak{D}\left(B_{\lambda}\right)=\mathfrak{D}\left(B_{\mu}\right)$,

$$
\mathfrak{D}\left(B_{\lambda} B_{\mu}\right)=\mathfrak{D}\left(B_{\mu}^{2}\right)=\mathfrak{D}((\mu I+T))=\mathfrak{D}(T)=\mathfrak{D}\left(B_{\mu} B_{\lambda}\right) .
$$

Because $Y_{\lambda}^{2}$ and $Y_{\mu}^{2}$ commute, Theorem 4 shows that $Y_{\lambda} Y_{\mu}=Y_{\mu} Y_{\lambda}$ and it follows that $B_{\lambda} B_{\mu}=B_{\mu} B_{\lambda}$.

If $0<\lambda<\mu$ the argument preceding (5) gives

$$
0 \leqq(\lambda I+T) Y_{\mu}^{2} \leqq(\mu I+T) Y_{\mu}^{2}=I .
$$

Hence, by (5), $Y_{\mu} \leqq Y_{\lambda}$. Thus $Y_{\lambda}-Y_{\mu}$ and $Y_{\mu}$ are commuting positive operators and $Y_{\lambda} Y_{\mu}-Y_{\mu}=Y_{\mu}\left(Y_{\lambda}-Y_{\mu}\right) \geqq 0$. Now, if $x \in \mathfrak{D}(T)$, $x=Y_{\lambda} Y_{\mu} w=Y_{\mu} Y_{\lambda} w$ for some $w$ in $\mathfrak{g}$ and

$$
\begin{aligned}
\left(B_{\lambda} B_{\mu} x, x\right) & =\left(B_{\lambda} B_{\mu} Y_{\mu} Y_{\lambda} w, Y_{\lambda} Y_{\mu} w\right) \\
& =\left(w, Y_{\lambda} Y_{\mu} w\right) \\
& \geqq\left(w, Y_{\mu}^{2} w\right) \\
& =\left(B_{\lambda} Y_{\lambda} Y_{\mu} w, B_{\lambda} Y_{\lambda} Y_{\mu} w\right) \\
& =\left(B_{\lambda}^{2} x, x\right) .
\end{aligned}
$$

THEOREM 9. Write $\mathfrak{D}=\mathfrak{D}\left(B_{1}\right)=\mathfrak{R}\left(\left\{(I+T)^{-1}\right\}^{\frac{1}{2}}\right)$. For each $x$ in $\mathfrak{D}$, $B_{\lambda} x$ tends to a limit as $\lambda \rightarrow 0+0$. The operator $B$ determined by $\mathscr{D}(B)=\mathscr{D}$, $B x=\lim _{\lambda \rightarrow 0+0} B_{\lambda} x$ is self-adjoint and positive and $B^{2}=T$.

Proof. Suppose $0<\lambda<\mu$ and let $x \in \mathfrak{D}(T)$. By Lemmas 8 and 7 


$$
\begin{aligned}
\left\|B_{\mu} x-B_{\lambda} x\right\|^{2} & =\left(B_{\mu}^{2} x, x\right)-2\left(B_{\lambda} B_{\mu} x, x\right)+\left(B_{\lambda}^{2} x, x\right) \\
& \leqq\left(B_{\mu}^{2} x, x\right)-2\left(B_{\lambda}^{2} x, x\right)+\left(B_{\lambda}^{2} x, x\right) \\
& =(\mu-\lambda)\|x\|^{2} .
\end{aligned}
$$

Suppose $x \in \mathfrak{D}$, because $B_{\mu}$ is self-adjoint, Lemma 2 (iii) shows there exists a sequence $\left(x_{n}\right)$ in $\mathfrak{D}\left(B_{\mu}^{2}\right)=\mathfrak{D}(T)$ such that $x_{n} \rightarrow x$ and $B_{\mu} x_{n} \rightarrow B_{\mu} x$. By (7),

$$
\begin{aligned}
\left\|B_{\lambda}\left(x_{n}-x_{m}\right)\right\| & \leqq\left\|B_{\mu}\left(x_{n}-x_{m}\right)\right\|+\left\|\left(B_{\mu}-B_{\lambda}\right)\left(x_{n}-x_{m}\right)\right\| \\
& \leqq\left\|B_{\mu}\left(x_{n}-x_{m}\right)\right\|+(\mu-\lambda)^{\frac{1}{2}}\left\|x_{n}-x_{m}\right\| \\
& \rightarrow 0(m, n \rightarrow \infty) .
\end{aligned}
$$

Hence, the sequence $\left(B_{\lambda} x_{n}\right)$ is convergent. Because $B_{\lambda}$ is self-adjoint, $B_{\lambda}$ is closed and $B_{\lambda} x_{n} \rightarrow B_{\lambda} x$. Thus,

$$
\begin{aligned}
\left\|B_{\mu} x-B_{\lambda} x\right\|^{2} & =\lim _{n \rightarrow \infty}\left\|B_{\mu} x_{n}-B_{\lambda} x_{n}\right\|^{2} \\
& \leqq \lim _{n \rightarrow \infty}(\mu-\lambda)\left\|x_{n}\right\|^{2} \\
& =(\mu-\lambda)\|x\|^{2} \quad(x \in \mathfrak{D}) .
\end{aligned}
$$

Relation (9) shows that, if $x \in \mathfrak{D}, B_{\lambda} x$ tends to a limit as $\lambda \rightarrow 0+0$. Define a (clearly linear) operator $B$ with domain $\mathfrak{D}$ by,

$$
B x=\lim _{\lambda \rightarrow 0+0} B_{\lambda} x \quad(x \in \mathfrak{D}) .
$$

By letting $\lambda \rightarrow 0+0$ in $(9)$, we have

$$
\left\|B_{\mu} x-B x\right\|^{2} \leqq \mu\|x\|^{2} \quad(x \in \mathscr{D})
$$

Now, suppose $x \in \mathfrak{D}(T)$; by Lemma 8 ,

$$
x \in \mathfrak{D}\left(B_{\lambda} B_{\mu}\right) \text { and } B_{\lambda} B_{\mu} x=B_{\mu} B_{\lambda} x \quad(\lambda>0, \mu>0) .
$$

Because $B_{\lambda} x \rightarrow B x, B_{\lambda} B_{\mu} x \rightarrow B B_{\mu} x(\lambda \rightarrow 0+0)$ and $B_{\mu}$ is closed we have $B_{\mu} B x=B B_{\mu} x(x \in \mathfrak{D}(T))$. Hence if $x \in \mathfrak{D}(T), B x \in \mathfrak{D}$ and $x \in \mathfrak{D}\left(B^{2}\right)$. Then,

By (10),

$$
\begin{aligned}
\left\|T x-B^{2} x\right\| & =\left\|-\mu x+B_{\mu}^{2} x-B B_{\mu} x+B_{\mu} B x-B^{2} x\right\| \\
& \leqq \mu\|x\|+\left\|\left(B_{\mu}-B\right) B_{\mu} x\right\|+\left\|\left(B_{\mu}-B\right) B x\right\| .
\end{aligned}
$$

$$
\begin{aligned}
\left\|T x-B^{2} x\right\| & \leqq \mu\|x\|+\mu^{\frac{1}{2}}\left\|B_{\mu} x\right\|+\mu^{\frac{1}{2}}\|B x\| \\
& \rightarrow 0(\mu \rightarrow 0+0, x \in \mathfrak{D}(T))
\end{aligned}
$$

This proves that $T \subseteq B^{2}$. 
If $x, y \in \mathfrak{D}$,

$$
(B x, y)=\lim \left(B_{\lambda} x, y\right)=\lim \left(x, B_{\lambda} y\right)=(x, B y) .
$$

Thus $B$ is symmetric, and $B^{2}=T$.

Suppose $\left(x_{n}\right)$ is a sequence in $\mathscr{D}$ such that $x_{n} \rightarrow x$ and $B x_{n} \rightarrow y$; take $\mu>0$. An argument, similar to the proof of (8) but based on (10) shows that the sequence $\left(B_{\mu} x_{n}\right)$ is convergent. Because $B_{\mu}$ is closed, $x \in \mathscr{D}$ and $B_{\mu} x_{n} \rightarrow B_{\mu} x(n \rightarrow \infty)$. Hence, by $(10)$,

$$
\begin{aligned}
\|y-B x\| & =\lim _{n \rightarrow \infty}\left\|B\left(x_{n}-x\right)\right\| \\
& \leqq \lim _{n \rightarrow \infty} \sup \left\{\left\|\left(B_{\mu}-B\right)\left(x_{n}-x\right)\right\|+\left\|B_{\mu}\left(x_{n}-x\right)\right\|\right. \\
& \leqq \lim _{n \rightarrow \infty}\left(\mu^{\frac{1}{2}}\left\|x_{n}-x\right\|+\left\|B_{\mu}\left(x_{n}-x\right)\right\|\right) \\
& =0 .
\end{aligned}
$$

This shows that $B$ is closed. Because we have already proved that $B$ is symmetric and $B^{2}=T$ it follows from Lemma 3 that $B$ is self-adjoint. Finally for $x \in \mathfrak{D}$,

$$
(B x, x)=\lim _{\lambda \rightarrow 0+0}\left(B_{\lambda} x, x\right) \geqq 0,
$$

which shows that $B$ is positive and completes the proof.

Before proving that $B$ is the unique positive self-adjoint square root of $T$ we need a result which, while important in its own right, is essentially a tricky technicality.

THEOREM 10. If $A$ is a closed operator which commutes with $T$ then $A$ commites with $B$.

Proof. Take $\lambda>0$. The hypothesis on $A$ shows that,

$$
\begin{aligned}
(\lambda I+T)^{-1} A & =(\lambda I+T)^{-1} A(\lambda I+T)(\lambda I+T)^{-1} \\
& \cong(\lambda I+T)^{-1}(\lambda I+T) A(\lambda I+T)^{-1} \\
& \cong A(\lambda I+T)^{-1} ;
\end{aligned}
$$

so, by Lemma $5, Y_{\lambda} A \subseteq A Y_{\lambda}$.

We now distinguish two cases

CASE (i). $A$ is bounded and $A T \subseteq T A$. In this case $\mathfrak{D}\left(Y_{\lambda} A\right)=\mathfrak{H}$ and hence $Y_{\lambda} A=A Y_{\lambda}$. Then

$$
\begin{aligned}
A B_{\lambda} & =A(\lambda I+T) Y_{\lambda} \\
& \subseteq(\lambda I+T) A Y_{\lambda} \\
& =(\lambda I+T) Y_{\lambda} A \\
& =B_{\lambda} A .
\end{aligned}
$$


Hence, for $x \in \mathscr{D}, A x \in \mathscr{D}$ and

$$
A B x=A \lim _{\lambda \rightarrow 0+0} B_{\lambda} x=\lim _{\lambda \rightarrow 0+0} A B_{\lambda} x=\lim _{\lambda \rightarrow 0+0} B_{\lambda} A x=B A x .
$$

Thus $A B \subseteq B A$ as required.

CASE (ii). $A$ is closed and $A T=T A$. This time we only have $Y_{\lambda} A \subseteq A Y_{\lambda}$ and hence

$$
B_{\lambda} A=(\lambda I+T) Y_{\lambda} A \subseteq(\lambda I+T) A Y_{\lambda}=A(\lambda I+T) Y_{\lambda}=A B_{\lambda} .
$$

Suppose $x \in \mathfrak{D}(A)$ and $A x \in \mathfrak{D}=\mathfrak{D}\left(B_{\lambda}\right)$. Then $B_{\lambda} x \in \mathfrak{D}(A)$ and $B_{\lambda} A x=A B_{\lambda} x$. Now $B_{\lambda} x \rightarrow B x$ and $A B_{\lambda} x=B_{\lambda} A x \rightarrow B A x \quad(\lambda \rightarrow 0+0)$. Because $A$ is closed, $B x \in \mathfrak{D}(A)$ and $A B x=\lim A B_{\lambda} x=B A x$. Hence, $B A \subseteq A B$.

Conversely, suppose $x \in \mathfrak{D}(A B)$. Take $\lambda>0$; then $A B x=A B B_{\lambda}^{2} Y_{\lambda}^{2} x$. Because $B_{\lambda}^{2}=\lambda I+T, B_{\lambda}^{2}$ commutes with $B$ and with $A$. Thus $A B x=B_{\lambda}^{2} A B Y_{\lambda}^{2} x$. This shows that $B Y_{\lambda}^{2} x \in \mathfrak{D}\left(B_{\lambda} A\right)=\mathfrak{D}(B A)$ and, because $B A \subseteq A B$,

$$
B A B Y_{\lambda}^{2} x=A B^{2} Y_{\lambda}^{2} x=A T Y_{\lambda}^{2} x=T A Y_{\lambda}^{2} x=B^{2} A Y_{\lambda}^{2} x .
$$

Hence, $Y_{\lambda}^{2} x \in \mathfrak{D}(B A)$ and $B A Y_{\lambda}^{2} x=A B Y_{\lambda}^{2} x$. We conclude that

$$
A B x=B_{\lambda}^{2} A B Y_{\lambda}^{2} x=B_{\lambda}^{2} B A Y_{\lambda}^{2} x=B A B_{\lambda}^{2} Y_{\lambda}^{2} x=B A x .
$$

Thus $A B \subseteq B A$ so that $A B=B A$ as required.

Note. Case (ii) includes the case of a bounded operator $A$ such that $A T=T A$. Under our convention (ii), $\mathfrak{D}(A)=\mathfrak{S}$ and hence, $A$ is closed and $A B=B A$.

The elegant use of the generalised Schwarz inequality in the next theorem was also pointed out to me by the referee of an earlier version of this paper.

THEOREM 11. The operator $B$ determined by Theorem 9 is unique. In other words, if $A$ is a positive self-adjoint operator such that $A^{2}=T$ then $A=B$.

Proof. Because $A T=A^{3}=T A$, Theorem 10 shows that $A B=B A$. Hence $\mathfrak{D}\left(T^{2}\right)=\mathfrak{D}\left(A^{2} B^{2}\right)=\mathfrak{D}(A B A B) \subseteq \mathfrak{D}(A B)$. Thus, if $x \in \mathfrak{D}\left(T^{2}\right)$ and $y=A x-B x$,

$$
(A+B) y=(A+B)(A-B) x=0 .
$$

Because $A$ and $B$ are positive, and

$$
0 \leqq(A y, y)+(B y, y)=((A+B) y, y)=0,
$$

we have $(A y, y)=0$. By the generalised Schwarz inequality $[4, \S 104]$

$$
|(A y, z)|^{2} \leqq(A y, y)(A z, z) \quad(z \in \mathfrak{D}(A)) .
$$


Hence $(A y, z)=0(z \in \mathfrak{D}(A)), A y=0$ and similarly $B y=0$. Then

$$
\begin{aligned}
\|(A-B) x\|^{2} & =(y, A x)-(y, B x) \\
& =(A y, x)-(B y, x) \\
& =0 \quad\left(x \in \mathfrak{D}\left(T^{2}\right)\right) .
\end{aligned}
$$

Thus $A x=B x(x \in \mathfrak{D}(T))$.

Let $B^{\prime \prime}$ and $B^{\prime}$ denote the restrictions of $B$ to $\mathscr{D}\left(T^{2}\right)$ and $\mathscr{D}(T)$ respectively and similarly for $A$. Now if $x, y \in \mathfrak{D}(T)$,

$$
\|B(x-y)\|^{2}=\left(B^{2}(x-y), x-y\right) \leqq\|T(x-y)\|\|x-y\| .
$$

It follows from Lemma 2 (iii) $\Gamma\left(B^{\prime \prime}\right)$ is dense in $\Gamma\left(B^{\prime}\right)$, and, in any case, $\Gamma(B)$ is the closure of $\Gamma\left(B^{\prime}\right)$. Thus $\Gamma(B)$ is the closure of $\Gamma\left(B^{\prime \prime}\right)$, similarly $\Gamma(A)$ is the closure of $\Gamma\left(A^{\prime \prime}\right)$. We have shown above that $\Gamma\left(A^{\prime \prime}\right)=\Gamma\left(B^{\prime \prime}\right)$; hence $\Gamma(A)=\Gamma(B)$ as required.

The graph juggling in the above proof is due to the referee. It replaces the author's original proof which was more complicated.

REMark. It $T$ is assumed to be bounded the proof of Theorem 11 collapses into a proof of uniqueness of $T^{\frac{1}{2}}$ which differs from that given in $[4, \S 104]$. The referee informs me it is essentially the same as given in [5].

Definition. If $T$ is a positive self-adjoint operator $T^{\frac{1}{2}}$ is the unique positive self-adjoint operator whose square is $T$.

\section{A decomposition theorem}

We shall prove the following theorem:

THEOREM 12. Let $A$ be a self-adjoint operator. Then there exist unique positive self-adjoint operators $A^{+}$and $A^{-}$such that: $A=A^{+}-A^{-}$; $\Re\left(A^{+}\right) \subseteq \mathfrak{R}\left(A^{-}\right) ; \Re\left(A^{-}\right) \subseteq \Re\left(A^{+}\right)$. Furthermore $A^{+}$and $A^{-}$commute with every bounded operator which commutes with $A$.

(For any operator $T, \mathfrak{R}(T)$ denotes the null space of $T$ ).

The remainder of this section consists of some definitions and three lemmas of which Theorem 12 is an immediate consequence.

We assume, throughout this section, that $A$ is a self-adjoint operator.

By Lemma 2(ii), $A^{2}$ is self-adjoint and positive. Accordingly define $|A|=\left(A^{2}\right)^{\frac{1}{2}}$.

LEMMA 13. $\mathfrak{D}(|A|)=\mathfrak{D}(A)$ and $\|A x\|=\||A| x\|(x \in \mathfrak{D}(A))$.

Proof. If $x \in \mathfrak{D}\left(A^{2}\right)=\mathfrak{D}\left(|A|^{2}\right)$,

$$
\|A x\|^{2}=\left(A^{2} x, x\right)=\left(|A|^{2} x, x\right)=\||A| x\|^{2} .
$$


By Lemma 2(iii) $\Gamma(|A|)$ is the closure of $\Gamma\left(|A|^{\prime}\right)$ where $|A|^{\prime}$ is the restriction of $|A|$ to $\mathscr{D}\left(|A|^{2}\right)=\mathscr{D}\left(A^{2}\right)$, and similarly for $\Gamma(A)$. The required results follow.

Now consider the operators $\frac{1}{2}(|A|+A)$ and $\frac{1}{2}(|A|-A)$; these have dense domain, $\mathscr{D}(A)$, and are clearly symmetric. Hence, $[4, \S 119]$, they have closed symmetric extensions $\left[\frac{1}{2}(|A|+A)\right]^{* *}$ and $\left[\frac{1}{2}(|A|-A)\right]^{* *}$.

Definition. $A^{+}=\left[\frac{1}{2}(|A|+A)\right]^{* *}, A^{-}=\left[\frac{1}{2}(|A|-A)\right]^{* *}$.

Lemma 14. (i) $A=A^{+-} A^{-}$, $|A|=A^{+}+A^{-}$;

(ii) $\Re\left(A^{+}\right) \subseteq \Re\left(A^{-}\right), \Re\left(A^{-}\right) \subseteq \mathfrak{N}\left(A^{+}\right)$;

(iii) $A^{2}=\left(A^{+}\right)^{2}+\left(A^{-}\right)^{2}$;

(iv) $A^{+}$and $A^{-}$are positive self-adjoint operators; with $A$.

(v) $A^{+}$and $A^{-}$commute with every bounded operator which commutes

Proof. (i) Clearly $A \subseteq A^{+}-A^{-}$and, because $A$ is self-adjoint and $A^{+}-A^{-}$is (obviously) symmetric, $A=A^{+}-A^{-}$. Similarly $|A|=A^{+}+A^{-}$.

(ii) Suppose $x \in \mathscr{D}\left(A^{2}\right)$. By Lemma 13, $|A| x \in \mathscr{D}(|A|)=\mathscr{D}(A)$ and $A^{+} x=\frac{1}{2}(|A|+A) x \in \mathfrak{D}(A) \subseteq \mathfrak{D}\left(A^{-}\right)$. Hence,

$$
\begin{aligned}
A^{-} A^{+} x & =\frac{1}{4}(|A|-A)(|A|+A) x \\
& =\frac{1}{4}(|A| A-A|A|) x .
\end{aligned}
$$

By Theorem 10, $|A| A=A|A|$ and hence,

$$
A^{-} A^{+} x=0 \quad\left(x \in \mathfrak{D}\left(A^{2}\right)\right) .
$$

Let $x \in \mathfrak{D}(A)$. By Lemma 2 (iii) there exists a sequence $\left(x_{n}\right)$ in $\mathscr{D}\left(A^{2}\right)$ such that $x_{n} \rightarrow x$ and $A x_{n} \rightarrow A x$. By Lemma 13, $|A| x_{n} \rightarrow|A| x$. Hence, $A^{+} x_{n} \rightarrow \frac{1}{2}(|A|+A) x=A^{+} x$. Because $A^{-}$is closed, $\mathfrak{R}\left(A^{-}\right)$is closed and $A^{+} x \in \mathfrak{R}\left(A^{-}\right)(x \in \mathfrak{D}(A))$. Finally, because $\mathfrak{R}\left(\frac{1}{2}(|A|+A)\right)$ is dense in $\Re\left(A^{+}\right)$ and $\mathfrak{N}\left(A^{-}\right)$is closed, we have $\Re\left(A^{+}\right) \subseteq \mathfrak{R}\left(A^{-}\right)$. To show that $\Re\left(A^{-}\right) \subseteq \mathfrak{N}\left(A^{+}\right)$ we can either argue similarly or use the obvious relations $A^{+}=(-A)^{-}$, $A^{-}=(-A)^{+}$.

(iii) If $x \in \mathfrak{D}\left(A^{2}\right)$, then, in particular,

$$
A^{+} x-A^{-} x=A x \in \mathfrak{D}\left(A^{+}\right) .
$$

By (ii), $A^{-} x \in \mathfrak{D}\left(A^{+}\right)$; hence, $A^{+} x \in \mathfrak{D}\left(A^{+}\right)$. Similarly $A^{-} x \in \mathfrak{D}\left(A^{-}\right)$and, by (ii)

$$
\begin{aligned}
A^{2} x & =A^{+}\left(A^{+} x-A^{-} x\right)-A^{-}\left(A^{+} x-A^{-} x\right) \\
& =\left(A^{+}\right)^{2} x+\left(A^{-}\right)^{2} x .
\end{aligned}
$$

Thus $A^{2} \subseteq\left(A^{+}\right)^{2}+\left(A^{-}\right)^{2}$. Because $A^{2}$ is self-adjoint and $A^{+}$and $A^{-}$are symmetric we have $A^{2}=\left(A^{+}\right)^{2}+\left(A^{-}\right)^{2}$, which is (iii). 
(iv) Let $x \in \mathscr{D}\left(A^{+*}\right)$. Recall, $[4, \S 117]$, that, because $A^{+}$is closed, $\Gamma\left(A^{+}\right)$is closed and its orthogonal complement in $\mathfrak{S} \times \mathfrak{S}$ is the set

$$
\left\{\left[A^{+*} u,-u\right]: u \in \mathscr{D}\left(A^{+*}\right)\right\} \text {. }
$$

Hence, there exist $y$ in $\mathscr{D}\left(A^{+}\right)$and $z$ in $\mathscr{D}\left(A^{+*}\right)$ such that:

in other words,

$$
\left[x, A^{+*} x\right]=\left[y, A^{+} y\right]+\left[A^{+*} z,-z\right]
$$

$$
x=y+A^{+*} z, A^{+*} x=A^{+} y-z .
$$

Because $A^{+}$is symmetric the first equation of (11) shows that $A^{+*} z \in \mathfrak{D}\left(A^{+*}\right)$. Hence, $A^{+*} x=A^{+} y+\left(A^{+*}\right)^{2} z$ and, (11) gives, $z=-\left(A^{+*}\right)^{2} z$. Suppose $v \in \mathfrak{D}\left(A^{-}\right)$; by (ii) $A^{-} v \in \mathfrak{R}\left(A^{+}\right)$and

$$
\left(A^{-} v, z\right)=\left(A^{-} v,-\left(A^{+*}\right)^{2} z\right)=\left(A^{+} A^{-} v,-A^{+*} z\right)=0 .
$$

Thus $z \in \mathfrak{R}\left(A^{-*}\right) \subseteq \mathfrak{D}\left(\left(A^{-*}\right)^{2}\right)$. Now, by (iii)

$$
\begin{aligned}
\left(A^{+*}\right)^{2}+\left(A^{-*}\right)^{2} & \subseteq\left(\left(A^{+}\right)^{2}\right)^{*}+\left(\left(A^{-}\right)^{2}\right)^{*} \\
& \subseteq\left(\left(A^{+}\right)^{2}+\left(A^{-}\right)^{2}\right)^{*} \\
& =\left(A^{2}\right)^{*} \\
& =A^{2} .
\end{aligned}
$$

Hence, $z \in \mathfrak{D}\left(A^{2}\right) \subseteq \mathfrak{D}\left(\left(A^{+}\right)^{2}\right)$; therefore $\left(A^{+*}\right)^{2} z=\left(A^{+}\right)^{2} z$ and

$$
0 \leqq\|z\|^{2}=\left(z,-\left(A^{+}\right)^{2} z\right)=-\left\|A^{+} z\right\|^{2} \leqq 0 .
$$

Because $z=0$, (11) shows that $x=y \in \mathfrak{D}\left(A^{+}\right)$and hence, $A^{+}$is self-adjoint. Now $A^{-}=(-A)^{+}$is also self-adjoint.

It is convenient, for later applications to give the proof that $A^{-}$is positive. Observe first that, because $A^{+}$is closed, $\mathfrak{R}\left(A^{+}\right)$is closed. Let $E$ be the orthogonal projection onto $\Re\left(A^{+}\right)$. Clearly $A^{+} E=0$ and,

$$
E A^{+}=E^{*} A^{+*} \cong(A+E)^{*}=0,
$$

so that $E A^{+}=0 \mid \mathfrak{D}\left(A^{+}\right)$. Because $\mathfrak{R}\left(A^{-}\right) \subseteq \mathfrak{R}\left(A^{+}\right), E A^{-}=A^{-}$; hence, $[4, \S 115]$, because $E$ is bounded,

$$
A^{-} E=A^{-*} E^{*}=\left(E A^{-}\right)^{*}=A^{-*}=A^{-} .
$$

Hence, if $x \in \mathfrak{D}(A)$,

$$
\begin{aligned}
\left(A^{-} x, x\right) & =\left(E A^{-} x, x\right) \\
& =\left(E A^{-} x, E x\right) \\
& =\left(A^{-} E x, E x\right) \\
& =\left(\left(A^{+}+A^{-}\right) E x, E x\right) \\
& =(|A| E x, E x) \\
& \geqq 0 .
\end{aligned}
$$


If $x \in \mathscr{D}\left(A^{-}\right)$there exists, by Lemma 1 , a sequence $\left(x_{n}\right)$ in $\mathscr{D}(A)$ such that $x_{n} \rightarrow x$ and $A^{-} x_{n} \rightarrow A^{-} x$. Thus $0 \leqq \lim \left(A^{-} x_{n}, x_{n}\right)=\left(A^{-} x, x\right) ; A^{-}$is positive and similarly, $A^{+}$is positive.

(v) Suppose $B$ is bounded and $B A \subseteq A B$. By Theorem $10, B|A| \subseteq|A| B$. Thus

$$
B A^{+} x=\frac{1}{2} B(|A|+A) x=A^{+} B x \quad(x \in \mathscr{D}(A)) .
$$

If $x \in \mathfrak{D}\left(A^{+}\right)$, the usual closure argument shows that $B x \in \mathfrak{D}\left(A^{+}\right)$and $B A^{+} x=A^{+} B x$. Thus $B A^{+} \subseteq A^{+} B$ as required.

Lemma 15. Suppose $B$ and $C$ are positive self-adjoint operators such that: $\mathfrak{R}(B) \subseteq \mathfrak{R}(C) ; \mathfrak{R}(C) \leqq \mathfrak{N}(B) ; A=B-C$. Then, $B=A^{+}, C=A^{-}$.

Proof. We begin by showing that $B+C$ is self-adjoint. The same argument used to prove Lemma 14(iii) shows that

$$
A^{2}=B^{2}+C^{2}=(B \pm C)^{2} .
$$

Because $B+C$ is clearly symmetric it is now sufficient, by Lemma 3 , to prove that $B+C$ is closed. If $x \in \mathfrak{D}(B+C)=\mathfrak{D}(B-C)=\mathfrak{D}(A)$,

$$
\begin{aligned}
\|(B \pm C) x\|^{2} & =\|B x\|^{2} \pm(B x, C x) \pm(C x, B x)+\|C x\|^{2} \\
& =\|B x\|^{2} \pm(x, B C x) \pm(B C x, x)+\|C x\|^{2} \\
& =\|B x\|^{2}+\|C x\|^{2} .
\end{aligned}
$$

Hence, if $\left(x_{n}\right)$ is a sequence in $\mathscr{D}(A)$ such that $x_{n} \rightarrow x$ and $(B+C) x_{n} \rightarrow y$, it follows that $\left(B x_{n}\right)$ and $\left(C x_{n}\right)$ are both Cauchy sequences. Because $B$ and $C$ are both closed, $x \in \mathfrak{D}(B+C), B x_{n} \rightarrow B x$ and $C x_{n} \rightarrow C x$. Thus $y=\lim (B+C) x_{n}=B x+C x$ and $B+C$ is closed.

It is clear that $B+C$ is positive so, by Theorem $11, B+C=|A|$. Thus,

$$
\frac{1}{2}(|A|+A)=\frac{1}{2}(B+C+B-C) \subseteq B .
$$

Because $B$ is self-adjoint,

$$
B \supseteq\left[\frac{1}{2}(|A|+A)\right]^{* *}=A^{+}
$$

and, because $A^{+}$is self-adjoint, $B=A^{+}$. Similarly $C=A^{-}$, which completes the proof.

\section{The spectral theorem for self-adjoint operators}

Throughout this section $A$ is a self-adjoint operator, projection means orthogonal projection, and statements about convergence of projections refer to strong convergence.

Definitron. A spectral family is a family $\{F(\lambda):-\infty<\lambda<\infty\}$ of projections such that 
(i) $F(\lambda) \leqq F(\mu) \quad(\lambda<\mu)$;

(ii) $F(\lambda+0)=F(\lambda)$;

(iii) $F(\lambda) \rightarrow 0 \quad(\lambda \rightarrow-\infty), \quad F(\lambda) \rightarrow I \quad(\lambda \rightarrow \infty)$.

Definition. For real $\lambda, E(\lambda)$ is the projection on $\mathfrak{N}\left((A-\lambda I)^{+}\right)$.

LEMMA 16. For each real $\lambda, E(\lambda)$ commutes with $A$ and with every bounded operator which commutes with $A$.

Proof. The proof that $A^{-}$is positive in Lemma 14 (iv) shows that

$$
\left\{\begin{array}{l}
0=(A-\lambda I)^{+} E(\lambda) \supseteqq E(\lambda)(A-\lambda I)^{+}=0 \mid \mathfrak{D}\left((A-\lambda I)^{+}\right), \\
(A-\lambda I)^{-} E(\lambda)=(A-\lambda I)^{-}=E(\lambda)(A-\lambda I)^{-} .
\end{array}\right.
$$

Hence $E(\lambda)(A-\lambda I) \leqq(A-\lambda I) E(\lambda)$ and $E(\lambda) A \subseteq A E(\lambda)$. Suppose $B$ is bounded and $B$ commutes with $A$. By Lemma 14 (v), $B$ commutes with $(A-\lambda I)^{+}$. Hence, by (12),

$$
0=B(A-\lambda I)+E(\lambda)=(A-\lambda I)+B E(\lambda) .
$$

Thus, $B E(\lambda)=E(\lambda) B E(\lambda)$. Because $B$ is bounded,

$$
\begin{aligned}
B^{*}(A-\lambda I)^{+} & \cong\left((A-\lambda I)^{+} B\right)^{*} \\
& \cong\left(B(A-\lambda I)^{+}\right)^{*} \\
& =(A-\lambda I)^{+} B^{*} .
\end{aligned}
$$

Hence, $B^{*} E(\lambda)=E(\lambda) B^{*} E(\lambda)$ and

as required.

$$
E(\lambda) B=\left(B^{*} E(\lambda)\right)^{*}=E(\lambda) B E(\lambda)=B E(\lambda),
$$

The aim now is to prove that $\{E(\lambda):-\infty<\lambda<\infty\}$ is a spectral family. It is convenient to do this in two stages.

Lemma 17. If $\lambda$ and $\mu$ are real with $\lambda<\mu$ then, $E(\lambda) \leqq E(\mu)$ and $A(E(\mu)-E(\lambda))$ is bounded and self-adjoint; furthermore,

$$
\lambda(E(\mu)-E(\lambda)) \leqq A(E(\mu)-E(\lambda)) \leqq \mu(E(\mu)-E(\lambda))
$$

in the usual ordering of bounded, self-adjoint operators and hence

$$
\|A(E(\mu)-E(\lambda))\| \leqq \max \{|\lambda|,|\mu|\} .
$$

Proof. By Lemma 16, $E(\lambda) E(\mu)=E(\mu) E(\lambda)$ and $E(\lambda)$ commutes with $A-\mu I$. By Lemma $14(\mathrm{v}), E(\lambda)$ commutes with $(A-\mu I)^{+}$and, by (12), $(A-\mu I)^{+} \supseteqq(I-E(\mu))(A-\mu I)$. Hence, if $x \in \mathfrak{D}(A)$ and $\lambda<\mu$,

$$
\begin{aligned}
(A-\mu I)+E(\lambda) x & =E(\lambda)\{I-E(\mu)\}(A-\mu I) x \\
& =\{I-E(\mu)\} E(\lambda)(A-\lambda I) x-(\mu-\lambda)\{I-E(\mu)\} E(\lambda) x \\
& =-\{I-E(\mu)\}(A-\lambda I)-E(\lambda) x-(\mu-\lambda)\{I-E(\mu)\} E(\lambda) x .
\end{aligned}
$$


Hence, because $E(\mu)$ commutes with $(A-\lambda I)^{-}$,

$$
\begin{aligned}
0 & \leqq\left((A-\mu I)^{+} E(\lambda) x, E(\lambda) x\right) \\
& =-\left(\{I-E(\mu)\}(A-\lambda I)^{-E} E(\lambda) x, E(\lambda) x\right)-(\mu-\lambda)(\{I-E(\mu)\} E(\lambda) x, E(\lambda) x) \\
& \leqq-(\mu-\lambda)\|\{I-E(\mu)\} E(\lambda) x\|^{2} \\
& \leqq 0 .
\end{aligned}
$$

Thus $(I-E(\mu)) E(\lambda) x=0(x \in \mathfrak{D}(A))$ and, because $\mathfrak{D}(A)$ is dense in $\mathfrak{F}$, $(I-E(\mu)) E(\lambda)=0$. That is $E(\lambda)=E(\mu) E(\lambda)$ and $E(\lambda) \leqq E(\mu)(\lambda<\mu)$. Because $E(\lambda) \leqq E(\mu), E(\mu)-E(\lambda)$ is a projection and certainly commutes with $A$. Hence, if $x \in \mathscr{D}(A)$ and $y=E(\mu) x-E(\lambda) x$, then $y=E(\mu) y$ and

Similarly,

$$
\begin{aligned}
(A(E(\mu)-E(\lambda)) x, x) & =(A y, y) \\
& =((A-\mu I) E(\mu) y, y)+\mu(y, y) \\
& =\left(-(A-\mu I)^{-} y, y\right)+\mu\|y\|^{2} \\
& \leqq \mu\|y\|^{2} \\
& =\mu((E(\mu)-E(\lambda)) x, x) .
\end{aligned}
$$

$$
(A(E(\mu)-E(\lambda)) x, x) \geqq \lambda\|(E(\mu)-E(\lambda)) x\|^{2}=\lambda((E(\mu)-E(\lambda)) x, x) .
$$

Now, write $M=\max \{|\lambda|,|\mu|\}$; by arguments based on $[3, \S 18$, Theorem 3$]$ it follows that

$$
\|A(E(\mu)-E(\lambda)) x\| \leqq M\|(E(\mu)-E(\lambda)) x\|(x \in \mathbb{D}(A)) .
$$

If $x \in \mathfrak{S}$, there exists a sequence $\left(x_{n}\right)$ in $\mathscr{D}(A)$ such that $x_{n} \rightarrow x$. Then, $(E(\mu)-E(\lambda)) x_{n} \rightarrow(E(\mu)-E(\lambda)) x$ and, because $A(E(\mu)-E(\lambda))$ is bounded on $\mathscr{D}(A)$, the sequence $\left(A\{E(\mu)-E(\lambda)\} x_{n}\right)$ is convergent. Because $A$ is closed, $(E(\mu)-E(\lambda)) x \in \mathfrak{D}(A)$. Thus $A(E(\mu)-E(\lambda))$ is defined everywhere and bounded and

Next,

$$
\|A(E(\mu)-E(\lambda))\| \leqq M=\max \{|\lambda|,|\mu|\} .
$$

Hence,

$$
A(E(\mu)-E(\lambda))=(E(\mu)-E(\lambda)) A(E(\mu)-E(\lambda)) .
$$

$$
\begin{aligned}
\{A(E(\mu)-E(\lambda))\}^{*} & \supseteqq(E(\mu)-E(\lambda)) A(E(\mu)-E(\lambda)) \\
& =A(E(\mu)-E(\lambda)) .
\end{aligned}
$$

Thus $A(E(\mu)-E(\lambda))$ is symmetric and, being everywhere defined, selfadjoint. Finally, the inequalities

$$
\lambda(\{E(\mu)-E(\lambda)\} x, x) \leqq(A\{E(\mu)-E(\lambda)\} x, x) \leqq \mu(\{E(\mu)-E(\lambda)\} x, x),
$$


which we have already proved for $x$ in $\mathscr{D}(A)$, extend, by continuity, to all $x$ in $\mathfrak{F}$. Thus,

$$
\lambda(E(\mu)-E(\lambda)) \leqq A(E(\mu)-E(\lambda)) \leqq \mu(E(\mu)-E(\lambda)),
$$

which completes the proof.

Corollary. If $x \in \mathscr{D}(A)$

$$
(A\{I-E(\lambda)\} x,\{I-E(\lambda)\} x) \geqq \lambda\|\{I-E(\lambda)\} x\|^{2} .
$$

Proof. Let $\mu \rightarrow \infty$ in the first inequality of (13).

Lemma 18. $\{E(\lambda):-\infty<\lambda<\infty\}$ is a spectral family.

Proof. Condition (i) of the requirements for a spectral family has been proved in Lemma 17.

To prove condition (ii) write $P=E(\lambda+0)-E(\lambda)$. $P$ is a projection and, because $E(\mu) \geqq E(\lambda+0) \geqq E(\lambda)(\mu>\lambda), P=(E(\mu)-E(\lambda)) P(u>\lambda)$ and $E(\lambda) P=0$. By (13),

$$
\begin{aligned}
0 & \leqq A(E(\mu)-E(\lambda))-\lambda(E(\mu)-E(\lambda)) \\
& \leqq(\mu-\lambda)(E(\mu)-E(\lambda)) ;
\end{aligned}
$$

and

$$
\|A(E(\mu)-E(\lambda))-\lambda(E(\mu)-E(\lambda))\| \leqq \mu-\lambda .
$$

By Lemma 17,

Hence,

$$
P x=(E(\mu)-E(\lambda)) P x \in \mathfrak{D}(A) \quad(x \in \mathfrak{S}) .
$$

$$
\|A P x-\lambda P x\|=\|(A-\lambda I)(E(\mu)-E(\lambda)) P x\| \leqq(\mu-\lambda) \| P x \quad(x \in \mathfrak{S}) .
$$

Letting $\mu \rightarrow \lambda+0$ we deduce, $A P x=\lambda P x$. Now,

Hence,

$$
(A-\lambda I)^{-} P x=(A-\lambda I)^{-} E(\lambda) P x=0 .
$$

$$
(A-\lambda I)^{+} P x=(A-\lambda I) P x=0 ;
$$

and $P x \in \mathfrak{R}\left((A-\lambda I)^{+}\right)$. Thus, $P x=E(\lambda) P x=0 \quad(x \in \mathfrak{Y})$. This shows that $E(\lambda+0)=E(\lambda)$ as required.

To show that $E(\lambda) \rightarrow 0(\lambda \rightarrow-\infty)$ write $E(-\infty)=\lim _{\lambda \rightarrow-\infty} E(\lambda)$. Let $x \in \mathfrak{D}(A)$; because $A$ is closed, the usual argument shows that $E(-\infty) x \in \mathscr{D}(A)$ and $A E(-\infty) x=\lim A E(\lambda) x=\lim E(\lambda) A x=E(-\infty) A x$. Write $y=E(-\infty) x$. For all $\lambda, y=E(\lambda) y$ and

$$
((A-\lambda I) y, y)=((A-\lambda I) E(\lambda) y, y)=-\left((A-\lambda I)^{-} y, y\right) \leqq 0 .
$$

Hence, $(A y, y) \leqq \lambda\|y\|^{2}$ for all $\lambda$. If $y \neq 0$ we obtain a contradiction by letting $\lambda \rightarrow-\infty$. Thus $E(-\infty) x=0(x \in \mathscr{D}(A))$ and, by continuity 
of $E(-\infty), E(-\infty)=0$. Similarly, if $E(\infty)=\lim _{\lambda \rightarrow \infty} E(\lambda)$, we have $E(\infty) x \in \mathfrak{D}(A)(x \in \mathfrak{D}(A)), E(\lambda) E(\infty)=E(\lambda)$ for all $\lambda$ and, if $y=(I-E(\infty)) x$ and $x \in \mathfrak{D}(A), y=(I-E(\lambda)) y$ and $((A-\lambda I) y, y)=\left((A-\lambda I)^{+} y, y\right) \geqq 0$. Hence, $E(\infty) x=x(x \in \mathscr{D}(A))$ and $E(\infty)=I$ as required.

We wish next to establish the formula

$$
A=\int_{-\infty}^{\infty} \lambda E(d \lambda) \text {, }
$$

for $A$. To do this we must first define the symbols used. For arbitrary real $a, b$ the integral $\int_{a}^{b} \lambda E(d \lambda)$ is a well defined Rieman-Stieltjes integral and defines a bounded operator on $\mathfrak{H}$, see for example $[4, \S 107]$. It is even true that the approximating sums converge uniformly to the integral. Because $A$ is not, in general, bounded we must think in terms of strong, rather than uniform, convergence. Accordingly we interpret the formula

$$
A=\int_{-\infty}^{\infty} \lambda E(d \lambda)
$$

as follows. The domain of $A$ is to be the set of $x$ in $\mathscr{H}$ such that $\int_{a}^{b} \lambda E(d \lambda) x$ tends to a limit as $a \rightarrow-\infty$ and $b \rightarrow \infty$; (this is clearly equivalent to: $\int_{-b}^{b} \lambda E(d \lambda) x$ tends to a limit as $\left.b \rightarrow \infty\right)$. Then, for $x \in \mathscr{D}(A)$,

$$
A x=\lim _{a \rightarrow-\infty, b \rightarrow \infty} \int_{a}^{b} \lambda E(d \lambda) x .
$$

This definition is clearly valid for an arbitrary spectral family and, because $E(b)-E(a)$ is easily seen to commute with $\int_{a}^{b} \lambda E(d \lambda)$, it follows that the definition gives rise to a densely defined operator.

THEOREM 19. There exists a spectral family, $\{E(\lambda):-\infty<\lambda<\infty\}$ such that, for all $\lambda, E(\lambda)$ commutes with $A$ and with every bounded operator which commutes with $A$ and

$$
A=\int_{-\infty}^{\infty} \lambda E(d \lambda)
$$

Proof. In view of Lemmas 17 and 18 it is only necessary to prove that $A=\int \lambda E(d \lambda)$.

The inequalities (13) show as in [4, § 107] that, if $a<b$,

$$
A(E(b)-E(a))=\int_{a}^{b} \lambda E(d \lambda) .
$$

Write $B=\int_{-\infty}^{\infty} \lambda E(d \lambda)$ and suppose $x \in \mathfrak{D}(B)$. Then

$$
\begin{aligned}
A(E(b)-E(a)) x & =\int_{a}^{b} \lambda E(d \lambda) x \\
& \rightarrow B x(a \rightarrow-\infty, b \rightarrow \infty) ;
\end{aligned}
$$

and, $(E(b)-E(a)) x \rightarrow x(a \rightarrow-\infty, b \rightarrow \infty)$. Because $A$ is closed, $x \in \mathscr{D}(A)$ and $A x=B x$. Thus $B \cong A$. Conversely, if $x \in \mathfrak{D}(A)$, 


$$
\begin{aligned}
\int_{a}^{b} \lambda E(d \lambda) x & =A(E(b)-E(a)) x \\
& =(E(b)-E(a)) A x \\
& \rightarrow A x(a \rightarrow-\infty, b \rightarrow \infty) .
\end{aligned}
$$

Thus $A \subseteq B$, which completes the proof.

THEOREM 20. If $\{F(\lambda):-\infty<\lambda<\infty\}$ is a spectral family such that

$$
A=\int_{-\infty}^{\infty} \lambda F(d \lambda)
$$

then $F(\lambda)=E(\lambda)(-\infty<\lambda<\infty)$.

Proof. We show that for real $\lambda, F(\lambda)$ is the projection on $\mathfrak{N}\left((A-\lambda I)^{+}\right)$. The case $\lambda=0$ is typical and notationally, simplest.

Define an operator $B$ as follows. $\mathfrak{D}(B)$ is the set of $x$ in $\mathfrak{S}$ such that $\int_{0}^{b} \lambda F(d \lambda) x$ tends to a limit as $b \rightarrow \infty$. For $x \in \mathscr{D}(B), B x=\lim _{b \rightarrow \infty} \int_{0}^{b} \lambda F(d \lambda) x$. Similarly define $C=-\int_{-\infty}^{0} \lambda F(d \lambda)$.

For $b>0, F(b) x \in \mathfrak{D}(B)$,

$$
B F(b) x=\int_{0}^{b} \lambda F(d \lambda) x
$$

and, if $x \in \mathscr{D}(B), B F(b) x \rightarrow B x(b \rightarrow \infty)$. Clearly $F(b) B \subseteq B F(b)$. Hence, if $x, y \in \mathfrak{D}(B)$,

$$
(B x, y)=\lim _{b \rightarrow \infty}(B F(b), y)=\lim _{b \rightarrow \infty}(x, B F(b) y)=(x, B y) .
$$

(here we use the known fact that $\int_{0}^{b} \lambda F(d \lambda)$ is self-adjoint). This shows that $B$ is symmetric. Let $x \in \mathfrak{D}(B), y \in \mathfrak{D}\left(B^{*}\right)$;

Thus,

$$
\left(x, F(b) B^{*} y\right)=(B F(b) x, y)=(x, B F(b) y) \text {. }
$$

$$
B F(b) y=F(b) B^{*} y \rightarrow B^{*} y \quad(b \rightarrow \infty) .
$$

This shows that $y \in \mathfrak{D}(B)$ and $B y=B^{*} y$. Thus $B$ is self-adjoint. Finally

$$
(B x, x)=\int_{0}^{\infty} \lambda(F(d \lambda) x, x) \geqq 0 \quad(x \in \mathfrak{D}(B)),
$$

and $B$ is positive. Similarly $C$ is self-adjoint and positive.

Next we show that $F(0)$ is the projection on $\mathfrak{R}(B)$. Clearly $B F(0)=0$. Suppose $B x=0$ and $\delta>0$,

$$
\begin{aligned}
0=(B x, x) & =\int_{0}^{\infty} \lambda(F(d \lambda) x, x)=\int_{0}^{\infty} \lambda\|F(d \lambda) x\|^{2} \\
& \geqq \int_{\delta}^{\infty} \lambda\|F(d \lambda) x\|^{2} \geqq \delta \int_{\delta}^{\infty}\|F(d \lambda) x\|^{2} \\
& =\delta\left(\|x\|^{2}-\|F(\delta) x\|^{2}\right) \\
& =\delta\|x-F(\delta) x\|^{2} .
\end{aligned}
$$


Hence $x=F(\delta) x(\delta>0)$ and, letting $\delta \rightarrow 0+0$ we obtain $x=F(0) x$. Finally,

Clearly $F(0) C=C$ and hence $\mathfrak{R}(C) \subseteq \mathfrak{N}(B)$. Similarly $\mathfrak{R}(B) \subseteq \mathfrak{R}(C)$.

$$
B-C=\int_{-\infty}^{\infty} \lambda F(\lambda \lambda)+0 \cdot(F(0)-F(0-0))=A .
$$

By Lemma 15, $B=A^{+}, C=A^{-}$. Hence, $F(0)=E(0)$ as required.

\section{Polar decomposition of a normal operator}

A closed operator $T$ is normal if $\mathfrak{D}(T)$ is dense in $\mathfrak{H}$ and $T T^{*}=T^{*} T$.

We begin with a lemma which is exercise 9 in [2, XII.9].

Lemma 21. (i) A closed operator $T$ is normal if and only if $T^{*}$ is normal;

(ii) $T$ is normal if and only if $\mathfrak{D}(T)=\mathfrak{D}\left(T^{*}\right)$ and $\|T x\|=\left\|T^{*} x\right\|$ $(x \in \mathfrak{D}(T))$;

(iii) a normal operator has no proper normal extension.

Proof. We content ourselves with remarking that Lemma 2(iii) is needed to prove the "only if" part of (ii).

Our next result is part of exercise 10 in [2, XII.9]. The proof we give is partly based on $[4, \S 110]$.

THEOREM 22. If $T$ is normal there exist a unique positive self-adjoint operator $|T|$ and a unitary operator $U$ such that $U|T|=|T| U=T . U$ is also uniquely determined if we require $U x=x(x \in \mathfrak{R}(T))$.

REMARK. A partial converse of this theorem, also given in exercise 10 of [2, XII.9] is the following:

if $T=U H \subseteq H U$ for some self-adjoint $H$ and unitary $U$, then $T$ is normal and $U H=H U$.

We shall not prove this converse.

Proof of Theorem 22. Let $|T|=\left(T^{*} T\right)^{\frac{1}{2}}$. By the argument of Lemma 13 , and by Lemma 21 (ii),

$$
\begin{aligned}
& \mathfrak{D}(T)=\mathfrak{D}\left(T^{*}\right)=\mathfrak{D}(|T|), \\
& \|T x\|=\left\|T^{*} x\right\|=\||T| x \mid\| \quad(x \in \mathfrak{D}(T)) .
\end{aligned}
$$

Write $\mathfrak{M}=(\Re(T))^{-}($the closure of $\mathfrak{R}(T))$. If $y \perp \mathfrak{M}, y \in \mathfrak{D}\left(T^{*}\right)$ and $T^{*} y=0$. Hence,

$$
\begin{aligned}
\mathfrak{M} & =(\Re(T))^{-}=\left(\Re\left(T^{*}\right)\right)^{-}=(\Re(|T|))^{-}, \\
\mathfrak{M}^{\perp} & =\mathfrak{R}(T)=\mathfrak{R}\left(T^{*}\right)=\mathfrak{N}(|T|) .
\end{aligned}
$$

If $x, y \in \mathfrak{D}(T)$ and $|T| x=|T| y$ then by $(16), x-y \in \mathfrak{R}(T)$ and $T x=T y$. 
Hence, for $x \in \mathscr{D}(T)$ we may define $U|T| x=T x$. This defines $U$ uniquely on $\Re(|T|)$ and, by (15), $U$ is isometric on $\Re(|T|)$ onto $\Re(T)$. Extend $U$ to $\mathfrak{M}$ by continuity giving an isometry of $\mathfrak{M}$ onto $\mathfrak{M}$. For $x \in \mathfrak{M}^{\perp}=\mathfrak{R}(T)$, define $U \boldsymbol{x}=\boldsymbol{x}$ and extend $U$ to $\mathfrak{H}$ by linearity. It is clear that $U$ is an isometric mapping of $\mathfrak{S}$ onto $\mathfrak{S}$. Hence, $U$ is unitary.

By definition of $U, T=U|T|$. Because $U$ is bounded, $T^{*}=|T| U^{*}$. Hence,

$$
|T|^{2}=T^{*} T=T T^{*}=U|T|^{2} U^{*}
$$

and $U|T|^{2}=|T|^{2} U$. By Theorem 10, $U|T|=|T| U$. Thus $T=U|T|=|T| U$ as required.

Finally, suppose $T=V H$ with $V$ unitary, $V x=x(x \in \mathfrak{R}(T))$ and $H$ self-adjoint and positive. Then, $T^{*} T=H V^{*} V H=H^{2}$. By Theorem 11, $H=|T|$ and it follows that $V=U$.

Corollary 1. If $T$ is $(\mathbf{1}-\mathbf{1})$ the conditions $T=U|T|$ with $U$ unitary determine $U$ uniquely.

CoRollary 2. $\Re(T)=\Re\left(T^{*}\right)=\Re(|T|)$.

Proof. Because $T=U|T|=|T| U$, it follows that

$$
U \mathfrak{D}(T)=\mathfrak{D}(T)=U^{2} \mathfrak{D}(T) \text {. }
$$

Hence,

$$
T x=|T|(U x)=T^{*}\left(U^{2} x\right) \quad(x \in \mathscr{D}(T)) .
$$

We close with a result which is required in [1].

THEOREM 23. Suppose that $\mathfrak{H}$ is complex, $T$ is normal and, for real $\lambda$ $E(\lambda)$ is the null space projection of $(|T|-\lambda I)^{+}$; then $E(\lambda)$ commutes with $T$ and $T E(\lambda)$ is a bounded normal operator with $\|T E(\lambda)\| \leqq \lambda$. Furthermore,

$$
\{T E(\lambda)\}^{*}=T^{*} E(\lambda), \quad|T E(\lambda)|=|T| E(\lambda) .
$$

Proof. Because $|T|$ is positive $E(\lambda)=0$ if $\lambda<0$. Hence, if $\lambda>0$,

$$
|T| E(\lambda)=|T|\left(E(\lambda)-E\left(-\frac{1}{2} \lambda\right)\right)
$$

and it follows from Lemma 17 that $|T| E(\lambda)$ is a bounded self-adjoint operator and $\||T| E(\lambda)\| \leqq \lambda$. If $\lambda=0,|T| E(\lambda)=0$ by definition. It follows from (15) that $T E(\lambda)$ and $T^{*} E(\lambda)$ are both bounded with norm less than or equal to $\lambda$. Because, by Theorem $22, T=U|T|=|T| U$ with $U$ unitary it follows from Lemma 16 that $E(\lambda)$ commutes with $|T|$ and with $U$ and hence with $T$ (and $T^{*}$ ). Now

$$
(T E(\lambda))^{*} \supseteq E(\lambda) T^{*} \text { and } E(\lambda) T^{*} \subseteq T^{*} E(\lambda) .
$$

Because $\mathfrak{D}\left(T^{*}\right)$ is dense it follows that $(T E(\lambda))^{*}=T^{*} E(\lambda)$ and (15) shows that $\|T E(\lambda) x\|=\left\|T^{*} E(\lambda) x\right\| \quad(x \in \mathfrak{S})$. Thus $T E(\lambda)$ is normal and (17) follows. 


\section{References}

[1] S. J. Bernau, 'The spectral theorem for unbounded normal operators', Pacific J. Math. 19 (1966), $391-406$.

[2] N. Dunford and J. T. Schwartz, Linear operators, Part II, (Interscience, New York, 1963).

[3] P. R. Halmos, Introduction to Hilbert space and the theory of spectral multiplicity (2nd. edition, Chelsea, New York, 1957).

[4] F. Riesz and B. Sz. Nagy, Functional Analysis, (Ungar, New York, 1955).

[5] B. Sz. Nagy, Spektraldarstellung linearer Transformationen des Hilbertschen Raumes (Ergebnisse d. Math., Band 5, Springer, Berlin, 1942).

[6] A. Wouk, 'A note on square roots of positive operators', SIAM Rev. 8 (1966), 100-102.

University of Otago

Dunedin, New Zealand 